\title{
Jimmie D. Lawson on the occasion of his 75th birthday
}

\section{Karl Heinrich Hofmann ${ }^{1,2}$}

Received: 21 April 2018 / Accepted: 21 April 2018 / Published online: 6 June 2018

C Springer Science+Business Media, LLC, part of Springer Nature 2018

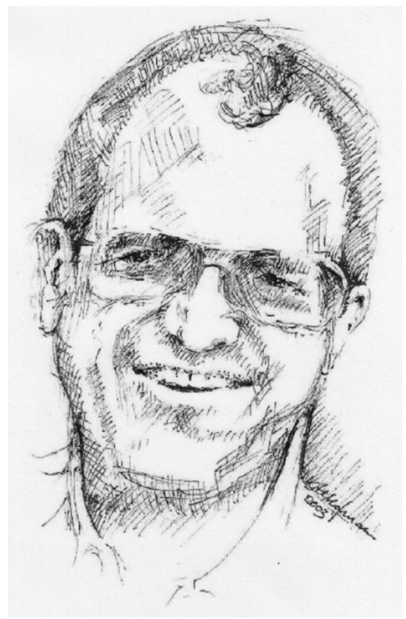

JIMMIE DON LAWSON was born on December 6, 1942 in Waukegan (Illinois). He attended Harding College in Searcy (Arkansas) and proceeded to study mathematics at the University of Tennessee in Knoxville. There he obtained his doctorate under the direction of DENNISON ROBERT BROWN in the year of 1967 with a dissertation

Communicated by László Márki.

$凶 \quad$ Karl Heinrich Hofmann

hofmann@mathematik.tu-darmstadt.de

1 Fachbereich Mathematik, Technische Universität Darmstadt, Schlossgartenstr. 7, 61289 Darmstadt, Germany

2 Department of Mathematics, Tulane University, New Orleans, LA 70118, USA 
entitled "Vietoris Mappings and Embeddings of Topological Semigroups". He moved on to Louisiana State University in Baton Rouge where he was appointed Assistant Professor in 1968.

He remained faithful to this institution, moving through the ranks until his career was crowned by his appointment to a Boyd Professorship in 1999; this title is the most prestigious one that LSU awards to select distinguished faculty members in any field of scholarship.

JIMMIE LAWSON's dissertation adviser DENNY BROWN was himself a student of ROBERT JACOB KOCH of Louisiana State University, through whom this institution had become in mathematics one of the centers of the study of topological semigroups, another being Tulane University in New Orleans, where KоCH acquired his degree in 1953 with a dissertation on "Topological Semigroups" under the direction of ALEXANDER DONIPHAN WALLACE, who had been at the core of the school of topological semigroups in the South of the United States in the fifties and sixties (see [6]). Equivalent to this fertile ground of topological algebra was the purely algebraic theory of semigroups promoted by ALFRED HOBLITZELLE CLIFFORD, whose two volume book with GORDON B. PRESTON on the algebra of semigroups was a landmark at that time [1]. The journal Semigroup Forum was founded by CLIFFORD, HOFMANN and MOSTERT at Tulane University with much support from the semigroup community in the late sixties, a journal to which Jimmie D. Lawson was to contribute so much both as an author and later as an editor through the years.

It was this mathematical environment that he came to join when he arrived at LSU. His originality and creativity as a prolific writer almost instantly influenced the field. I became familiar with Jimmie Lawson's professional impact at an early stage through common interests leading to coauthorships of a considerable number of articles and several books and common editorial tasks in collections and journals. This may make the reader understand the enthusiasm I feel towards JIMMIE LAWSON as a teacher, mathematician, administrator, as a colleague, and as a friend. His career spanned 50 years of excellence in research and academic service. He has served as Chairman of the large mathematics department of Louisiana State University. The mathematical community at large has always recognized his contributions. Prior to his appointment to a Boyd Professorship at LSU, he was the recipient of a fellowship of the Alexander von Humboldt-Foundation in 1980 to work at the University of Technology at Darmstadt, and a few years later the Deutsche Forschungsgemeinschaft (the analog in Germany of the National Science Foundation in the US) funded a Visiting Full Professorship for him at that University in 1992. In 2002 he returned as a Senior Humboldt Fellow. To many working mathematicians JIMMIE LAWSON is known for his editorial work on various levels. He was Chairman of the Board of Editors of the journal "Semigroup Forum" (Springer New York etc.) from 1992 to 1998 and continued as Executive Editor and Council Member of the Journal. He is an Editor of Topology and its Applications since 2015 and was Editor of the "Journal of Lie Theory" [Heldermann Verlag Lemgo (Germany)]. He was in high demand as an international outside referee of dissertations and served on the consulting boards of regular conferences (such as for instance the Summer Conferences on General Topology and its Applications Advisory Committee). 
It is not surprising that a scholar of JIMMIE LAWSON's originality and breadth also had a distinguished career of excellence as a teacher in undergraduate courses as well as graduate courses and advanced seminars alike. Around the globe JIMMIE LAWSON has always been a coveted colloquium speaker and conference participant on account of his outstanding talent of exposition which makes even very complicated matters appear clear and easy.

His Visiting Professorship at the Darmstadt Institute of Technology during the academic year 1992-1993 created an aftermath of several master's theses being written even after he left — so much did he captivate his audience by his presentation in a course on topological dynamics and chaos theory!

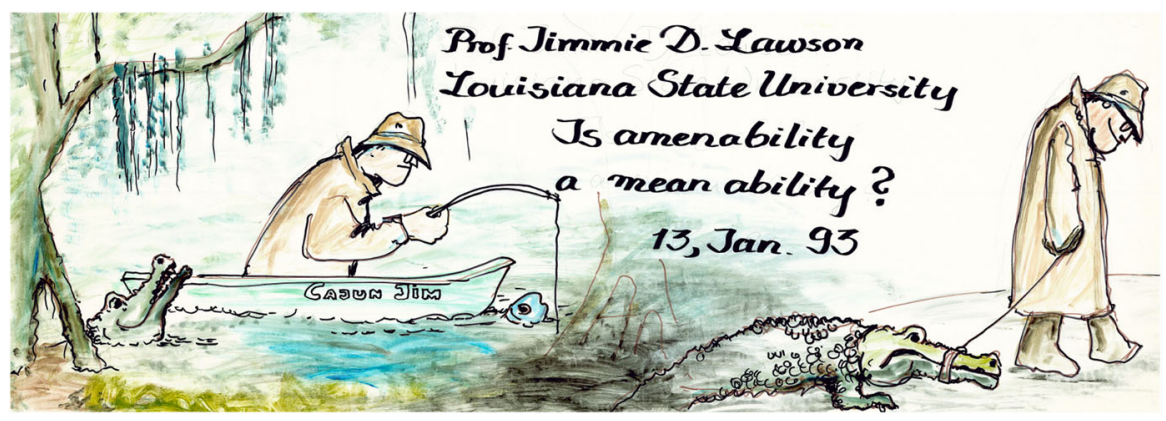

The Darmstadt Colloquium Poster of Jimmie's Lecture in 1993

JIMMIE LAWSON's mathematical contributions range through an amazingly wide circuit, beginning with topological semigroups as a root of so much that had caught his interests through the times, reaching from algebraic topology, through the theory of continuous lattices and posets (an area with important applications to theoretical computer science such as the denotational semantics of programming languages), through the linear algebra of matrix theory and positivity, all the way to the theory of Lie groups, mainly in the context of convexity. In each one of his areas JIMMIE LAWSON has made incisive contributions that have left his marks on these areas, forcing authors to refer to his work time and again. Many results of his research bear his name in the text book and monograph literature as well as in the journal articles in the respective specialties. A reference to "Lawson's Theorem" tends to be so common that experts equally versed in all of his fields sometimes need a pause to sort out exactly which one is meant right now. Typically, the introduction of a seminal monograph by NEEB [23] cites LAWSON's results prominently even though the core topic of the book is not exactly in any of LAWSON's specialties.

In Semigroup Forum, a tribute to JIMMIE DON LAWSON and his work should remind readers of at least a small selection of the details of his mathematical profile and comment how they relate to semigroup theory.

Lawson Semilattices In his dissertation, JIMMIE LAWSON dealt with compact semilattices (with identity, say) and characterized those which allowed enough continuous homomorphisms into the unit interval to separate points [15]. Workers in the area of topological groups will notice an analogy to compact abelian groups and continuous homomorphisms into the circle groups (called characters) (see e.g. [14]). At first sight, this category of compact semilattices together with its morphisms looks like 
yet another special topic devised for the purpose of a doctoral thesis. Yet, amazingly, it was exactly that class which was very shortly thereafter recognized to be identical with DANA S. SCOTT's continuous lattices which were discovered rather precisely at the time that LAWSON worked on his semilattices. Indeed when a continuous lattice is endowed with a certain compact Hausdorff topology now called Lawson topology it turns out to be a compact Lawson semilattice, and conversely if the topology of a Lawson semilattice is coarsened appropriately it is a continuous lattice.

Continuous lattices and posets In the course of his productive life, LAWSON contributed steadily to the theory of continuous lattices and posets, and its links with semigroup theory, in particular through his collaboration in writing the crucial monograph becoming known as "The Compendium" [2], and later its enlarged and updated version [3]. He had five coauthors in writing these often cited works; among these one notes other former editors of Semigroup Forum, namely, KLAUS KEIMEL, MIKE MisLOVE and myself. We all deeply deplored KLAUS KEIMEL's passing in 2017 [11]. JIMMIE LAWSON wrote and coauthored numerous significant papers in the area. For instance he developed the spectral theory of distributive continuous lattices with the result that one understood that distributive continuous lattices are (dually) equivalent to locally compact sober $T_{0}$-spaces [7].

Algebraic topology In algebraic topology, among a variety of topics, he participated in the creation and discussion of a definitive theory of intrinsically defined boundary points, the so-called peripheral points of a topological space. One of their applications in [22] was that all invertible elements in a compact topological semigroup had to be peripheral points.

Continuous posets As the interest of people concerned with the application of partially ordered sets (posets) to formal theories of computation shifted from lattices to more general structures, JIMMIE LAWSON established that the poset of SCOTT open filters on a continuous poset is a continuous poset and thereby established a perfect duality theory for continuous posets (now called Lawson duality): see [3] for this context. This line of research yielded the insight that the poset $Q(X)$ of compact saturated sets of a locally compact $T_{0}$-space $X$ is the Lawson dual of the continuous lattice $O(X)$ of open sets which - as we recall from above - is the most general distributive continuous lattice possible. (This result is now in the literature as HOFMANN-MisLOVE Theorem.)

Polish spaces In the general context of the foundations of analysis pursued by ABBAS EDALAT of Imperial College in London JIMMIE LAWSON contributed a much cited result to the field of continuous posets which, in some sense is parallel for Polish spaces to what the preceding set-up does for locally compact spaces [20]. This result associates with any continuous DCPO (i.e. "directed complete poset") with a countable basis for its open sets a Polish space, namely, the space of maximal points in the poset. Conversely, every Polish space is obtained in such a fashion. This is another result relating important classes of topological spaces with order structures, which themselves, as we have seen, are close neighbors to topological semigroups.

Compact semitopological semigroups A semigroup with a topology is called semitopological if all right and left translations are continuous selfmaps. Such semigroups frequently occur as operator semigroups on some Banach space with the weak operator topology. Here we encounter again a result called LAwsON's Theorem. It was he who 
established that the multiplication on a locally compact semitopological semigroup $S$ is jointly continuous in every pair $(x, y) \in S \times S$ one of whose members is invertible in the semigroup [16]. This includes ELLIS's Theorem saying that a group supporting a locally compact topology for which multiplication is separately continuous is already a topological group. LAWSON's contribution attained considerable significance in the area of dynamical systems and their ergodic theory [17].

Lie semigroups JIMMIE LAWSON was one of the founders of the entire theory as is documented in numerous articles (e.g. [8-10]), the first basic monographs on the field [4] and three collections of surveys $[5,12,13]$. In many circumstances one encounters subsemigroups of Lie groups. In nonlinear systems and control theory on Lie groups or homogeneous spaces, multiplicative subsets play a role in which inversion is partially possible at best. In the study of partially ordered manifolds (such as for instance a pseudo-Riemannian manifold with a Lorentzian metric) and of partially ordered symmetric spaces semigroups of Lie groups arise naturally. In the analytic extension of a unitary representation of a Lie group, the domain of definition frequently turns out to be a semigroup containing the given Lie group and being contained in a bigger Lie group. An example is the semigroup of all those Möbius transformations of the complex plane which respect the upper half-plane. Semigroups of this sort were discovered and discussed by OL'SHANSKII (cf. [19]). Coherent literature on the subject of semigroups in Lie groups exists since the eighties of the last century. Among the authors promoting the subject vigorously, JIMMIE LAWSON was one of the prominent pioneers as is documented in the standard sources now extant in the field (see e.g. $[4,5,12,13,18,21])$. In his contribution in Crelle's Journal [19] he succeeded in giving a characterisation of those closed subsemigroups of a Lie group which are of the OL'SHANSKI type. In pursuing this subject, JIMMIE LAWSON invested his profound insight into presenting to the research community in this area a very attractive geometric theory of semigroups, called MöBIUS semigroups [21].

In the theory of Lie semigroups as well as in so many other areas LAWSON's research is seminal to a field and spawns a whole generation of research. The clarity of thought and exposition in his work has served as a model to colleagues and students alike. He has the gift of subjecting a problem to an analysis which will rid it of all unnecessary details and at the same time find answers at the level of greatest possible generality on which the solution persists henceforth.

JIMMIE LAWSON celebrated his 75th birthday in the month of December of last year. On this occasion my tribute to him and his work concludes with my expression of gratitude that our professional biographies could be intertwined on two continents so firmly for many decades. Engraved in our memories are these days when Hurricane Katrina devastated New Orleans in August 2005. My wife Isolde and I fled from the approaching storm upriver in the company of our colleague WALTER MICHAELIS from the University of New Orleans. At that point in time we were uprooted refugees who were most warmly received by Janie and Jimmie Lawson in their home for several weeks in Baton Rouge until it was possible and safe to return to the barely functional remnant of our city. 


\section{References}

1. Clifford, A.H., Preston, G.B.: The Algebraic Theory of Semigroups. Mathematical Surveys, vol. 7. American Mathematical Society, Providence (1961)

2. Gierz, G., Hofmann, K.H., Lawson, J.D., Keimel, K., Mislove, M.W., Scott, D.S.: A Compendium of Continuous Lattices, $\mathrm{xx}+371 \mathrm{pp}$. Springer, New York (1980)

3. Gierz, G., Hofmann, K.H., Lawson, J.D., Keimel, K., Mislove, M.W., Scott, D.S.: Continuous Lattices and Domains, Encyclopedia of Mathematics and Its Applications, vol. 93, xxxvi+591pp. Cambridge University Press, Oxford (2003)

4. Hilgert, J., Hofmann, K.H., Lawson, J.D.: Lie Groups, Convex Cones, and Semigroups, xxxiii+645pp. Oxford University Press, Oxford (1989)

5. Hilgert, J., Lawson, J.D., Neeb, K.-H., Vinberg, E.B. (eds.): Positivity in Lie Theory: Open Problems. De Gruyter Expositions in Mathematics, vol. 26. de Gruyter Verlag, Berlin (1998)

6. Hofmann, K.H., Koch, R.J., Mostert, P.S.: Alexander Doniphan Wallace on his 68th birthday. Semigroup Forum 7, 10-31 (1973)

7. Hofmann, K.H., Lawson, J.D.: The spectral theory of continuous lattices. Trans. Am. Math. Soc. 246, 285-310 (1978)

8. Hofmann, K.H., Lawson, J.D.: The local theory of semigroups in nilpotent Lie groups. Semigroup Forum 23, 343-357 (1981)

9. Hofmann, K.H., Lawson, J.D.: On Sophus Lie's fundamental theorems I. Indag. Math. 45, 453-466 (1983)

10. Hofmann, K.H., Lawson, J.D.: On Sophus Lie's fundamental theorems II. Indag. Math. 46, 255-265 (1984)

11. Hofmann, K.H., Lawson, J.D.: In memoriam Klaus Keimel, 1939-2017. Semigroup Forum (2018). https://doi.org/10.1007/s00233-018-9929-2

12. Hofmann, K.H., Lawson, J.D., Pym, J.S. (eds.): The Analytical and Topological Theory of Semigroups. De Gruyter Expositions in Mathematics, vol. 1. de Gruyter Verlag, Berlin (1990)

13. Hofmann, K.H., Lawson, J.D., Vinberg, E.B. (eds.): Semigroups in Algebra, Analysis and Geometry. De Gruyter Expositions in Mathematics, vol. 20. de Gruyter Verlag, Berlin (1995)

14. Hofmann, K.H., Morris, S.A.: The Structure of Compact Groups, De Gruyter Studies in Mathematics, vol. 25, 3rd edn. de Gruyter, Berlin, xxii+924 pp (2013)

15. Lawson, J.D.: Topological semilattices with small semilattices. J. Lond. Math. Soc. 1, 719-724 (1969)

16. Lawson, J.D.: Joint continuity in semitopological semigroups. Ill. Math. J. 18, 275-285 (1972)

17. Lawson, J.D.: Additional notes on continuity in semitopological semigroups. Semigroup Forum 12, 443-453 (1976)

18. Lawson, J.D.: Historical links to a Lie theory of semigroups. J. Lie Theory 2, 263-278 (1992)

19. Lawson, J.D.: Polar and Ol'shanskiı̌ decompositions. J. Reine Angew. Math. 448, 191-219 (1994)

20. Lawson, J.D.: Spaces of maximal points. Math. Struct. Comput. Sci. 7, 543-555 (1997)

21. Lawson, J.D.: Semigroups in Möbius and Lorentzian geometry. Geom. Dedic. 70, 139-180 (1998)

22. Lawson, J.D., Madison, B.L.: Peripherality in semigroups. Semigroup Forum 1, 128-142 (1970)

23. Neeb, K.-H.: Holomorphy and Convexity in Lie Theory. De Gruyter Expositions in Mathematics, vol. 28. De Gruyter, Berlin (1999) 